\title{
Percepción de los pacientes trasplantados renales sobre la necesidad de información de sus donantes
}

\author{
Perception of kidney transplant patients of the need for information about their donors
}

\author{
Rubén Omar Schiavelli', Mariela Verónica Miranda², Ommi Acosta Sero ${ }^{3}$, Paula Morelli, $^{4}$ \\ Melisa Vázquez Melia ${ }^{5}$, Daniel Osvaldo Merino ${ }^{6}$
}

\section{Resumen}

Objetivo: Describir las percepciones de pacientes trasplantados renales sobre la necesidad de información acerca del donante y la potencial influencia del sexo y edad del mismo. Materiales y métodos: Estudio analítico, observacional, transversal y prospectivo de trasplantados renales mayores de 18 años con al menos I año de trasplante. Los datos se recolectaron por historia clínica y una encuesta semiestructurada. Resultados: Se analizaron 104 pacientes, $58 \%$ fueron mujeres, con una media de edad de 47 años y tiempo de trasplantados de 7 años. El porcentaje de pacientes a los que les interesaba conocer el sexo biológico y la edad del donante fue significativamente superior $(76 \% ; n=79 ; p<0,00 I)$. Solo el I $3 \%(n=\mid 4)$ refirió que elegiría el sexo y la edad del donante $(p<0,00 \mathrm{I})$, aunque una mayor proporción de pacientes $(78 \%)$ percibió que el sexo y la edad del donante podían influir en ellos $(\mathrm{p}<0,00 \mathrm{I})$. De los 79 pacientes que manifestaron interés en conocer el sexo y edad del donante, el $62 \%$ refirió que el motivo era agradecer, observándose diferencias con respecto a otros motivos $(p=0,0025)$. Conclusión: El agradecimiento dirigido a la persona de la cual solamente conoce el sexo y la edad permitiría al paciente trasplantado poder realizar una construcción imaginaria del donante.

Palabras Clave: Gratitud - Satisfacción con la vida - Receptor de trasplante renal - Aspectos psicológicos de los trasplantes.

\begin{abstract}
Objective: To describe the perceptions of renal transplant patients of the need for information about their donors and the potential influence of their donors' biological sex and age. Materials and methods: Analytical, observational, cross-sectional and prospective study. Kidney transplant patients over 18 years old who had been transplanted, at least, one year before were included. A semi-structured survey was conducted, and data were collected from medical records. Results: 104 patients were analyzed, 58\% $(n=60)$ were women; median age: 47 years; and median post-transplantation period: 7 years. Seventy-six percent $(n=79)$ expressed that they were interested in knowing their donors' biological sex and age $(p<0.001)$. When asked whether they would choose their donors'sex and age, only $13 \%(n=14)$ answered that they would $(p<0.001)$. When asked whether they felt that their donors' sex and age might have an influence on them, $78 \%(n=8 I)$ answered that they did not, while $22 \%$ answered that they did $(p<0.00 I)$. Of the 79 patients who
\end{abstract}

\footnotetext{
RECIBIDO 20/2/2021 - ACEPTADO 10/5/2021

'.Médico Especialista en Nefrología. Jefe de Nefrología y Trasplante Renal, Hospital General de Agudos “Dr. Cosme Argerich”. Ciudad de Buenos Aires, Argentina.

2.Licenciada en Psicología. Hospital General de Agudos “Dr. Cosme Argerich”. Ciudad de Buenos Aires, Argentina.

3.Magister en efectividad Clínica, Especialidad en estadística para Ciencias de la Salud, Licenciada y Profesora Universitaria en nutrición. Hospital General de Agudos "Dr. Cosme Argerich". Ciudad de Buenos Aires, Argentina.

4.Licenciada en Psicología. Hospital General de Agudos "Dr. Cosme Argerich". Ciudad de Buenos Aires, Argentina.

5.Licenciada en Psicología. Hospital General de Agudos "Dr. Cosme Argerich". Ciudad de Buenos Aires, Argentina.

6.Título Licenciado en Psicología. Hospital General de Agudos "Dr. Cosme Argerich". Ciudad de Buenos Aires, Argentina.
} 
expressed an interest in knowing their donors' biological sex and age, $62 \%(n=49)$ stated that appreciation was the reason for it $(p=0.0025)$. Conclusion: The appreciation towards that person about whom only sex and age are known would allow patients to imaginarily construct their donors.

Keywords: Gratitude - Satisfaction with life - Kidney recipient - Psychological aspects of transplants.

\section{Introducción}

En el trasplante de órganos o tejidos participan diferentes actores como son el donante, el receptor y el órgano a trasplantar. Esta práctica está atravesada por dimensiones biológicas, psicológicas y sociales. Sin embargo, existe una tendencia a enfocarse solo en la dimensión biológica del trasplante, probablemente a partir del legado de un discurso médico positivista que manifiesta que el éxito del mismo reside en la correcta asimilación y función del órgano o tejido (Fuica \& Palacios-Espinosa, 2016).

La realización de un trasplante exitoso devuelve, en principio, la salud al receptor, pero si se tiene en cuenta que salud es definida como el "estado de completo bienestar físico, mental y social y no meramente ausencia de enfermedad" (Organización Mundial de la Salud, 1946), aunque se logre un excelente funcionamiento del órgano trasplantado, si no se incluyen los aspectos psicológicos y sociales de los pacientes (Schiavelli, et al., 2011) se estará lejos de cumplir con ese precepto.

Desde la visión científica, los órganos cadavéricos para trasplante se consideran como repuestos que, bajo una técnica quirúrgica eficiente más un régimen inmunosupresor adecuado, pueden realizar su función dentro de los cuerpos de sus nuevos receptores. Sin embargo, para muchas personas la situación de recibir un órgano es más complicada y requiere de procesos psicológicos complejos (Vamos, 2010).

El objetivo del presente trabajo es describir las percepciones de los pacientes sometidos a un trasplante renal acerca de la necesidad de tener información sobre el donante y la potencial influencia del sexo biológico y la edad del mismo.

\section{Materiales y métodos}

Se desarrolló un estudio analítico, observacional, transversal y prospectivo, en el que se recolectaron datos de pacientes trasplantados renales que concurrieron a la consulta ambulatoria, desde marzo hasta octubre de 2019 en la División de Nefrología y Trasplante Renal del Hospital General de Agudos "Dr. Cosme Argerich", de la Ciudad de Buenos Aires, Argentina. El muestreo fue no probabilístico, por conveniencia.
Se incluyeron pacientes trasplantados renales estables, mayores de 18 años de ambos sexos biológicos y con al menos 1 año de trasplantados en seguimiento en nuestro consultorio.

Los datos se recolectaron a través de una encuesta semiestructurada con a) preguntas cerradas para las variables sociodemográficas y aquellas relacionadas con el trasplante renal (dichos datos se verificaron con lo registrado en las historias clínicas), y b) preguntas abiertas para explorar las percepciones de los pacientes trasplantados acerca de la necesidad de tener información sobre el donante.

Se analizaron:

a. variables sociodemográficas: sexo biológico (mujeres/hombres), edad (expresada en años cumplidos);

b. variables relacionadas con el trasplante renal: tiempo desde el trasplante (expresado en años), tiempo en lista de espera (expresado en años);

c. variables relacionadas con la necesidad de información sobre el donante: conocimiento sobre sexo biológico y la edad del donante, interés, elección e influencia del sexo biológico del donante.

\section{Análisis estadístico}

Las variables cualitativas se describieron a través de frecuencias absolutas y relativas, las variables numéricas a través de la mediana y el rango intercuartílico (RIC) ya que no presentaron distribución normal. Dicha normalidad se evaluó a través de métodos gráficos y analíticos (test de Shapiro-Wilk). Las variables cualitativas se analizaron a través del test de chi cuadrado. Para determinar si existían diferencias entre grupos con respecto a las variables cuantitativas se calculó el estadístico no paramétrico de Mann-Whitney. Para analizar diferencias en cuanto a lo referido por los pacientes sobre influencia y elección del sexo biológico del donante y motivo de la necesidad de tener información sobre este, se utilizó el test $\mathrm{Z}$ para diferencia de 2 proporciones. Los datos fueron procesados con el software estadístico R, versión 3.5.2 (The R Foundation for statistical computing ${ }^{\oplus} 2018$ ). Se consideró un nivel 
de significación $\alpha=0,05$. Con respecto a las respuestas obtenidas de las preguntas abiertas se extrajeron citas con los segmentos más significativos.

\section{Consideraciones bioéticas}

El presente estudio se adecuó a las normas internacionales de investigación de Helsinki de la Asociación Médica Mundial. Los pacientes firmaron un consentimiento informado autorizando el uso de los datos bajo la premisa de proteger la privacidad y confidencialidad de su información personal.

Los autores adhieren a la Declaración de Estambul sobre el tráfico de órganos y el turismo de trasplantes y al Documento de Aguascalientes sobre trasplante de órganos.

\section{Resultados}

La muestra quedó conformada por 104 pacientes, de los cuales el $58 \%(n=60)$ fueron mujeres, la mediana de edad fue 47 años (RIC: 35 - 57), mínimo 18 y máximo de 76 años. La mediana de tiempo desde el trasplante fue de 7 años (RIC: 3 - 10), mínimo 1 y máximo 21 años. La mediana de tiempo en lista de espera para el trasplante fue de 3 años (RIC: 1 - 5).

La mayoría de los pacientes conocían el sexo biológico $(86 \% ; \mathrm{n}=90)$ y la edad $(78 \% ; \mathrm{n}=81)$ de su donante. El $76 \%(n=79)$ refirió que le interesaba conocer el sexo biológico y la edad del donante, siendo dicho porcentaje significativamente superior al grupo de pacientes que refirió que no les interesaba $(\mathrm{p}<0,001)$.

Entre quienes manifestaron interés, la mayoría fueron mujeres (61\%), las que predominaron de manera significativa comparadas con los hombres $(\mathrm{p}=0,0068)$, además el tiempo de trasplante fue significativamente inferior entre quienes refirieron que les interesaba conocer el sexo biológico y la edad del donante (mediana: 6 años; RIC 2 - 9 años) comparados con quienes no (mediana: 7 años; RIC 6 - 12 años) ( $p=0,033)$. La edad y el tiempo en lista de espera no se asociaron con el interés en conocer el sexo biológico y la edad del donante ( $\mathrm{p}=0,704 ; \mathrm{p}=0,344$, respectivamente).

$\mathrm{Al}$ indagar sobre si los pacientes pudieran elegir el sexo y edad del donante, solo el 13\% $(\mathrm{n}=14)$ precisó que sí, observándose diferencias significativas comparados con el porcentaje que refirió que no lo elegiría. $(\mathrm{p}<0,001)$. Entre quienes preferían elegirlos $(\mathrm{n}=14)$, 9 eran mujeres y 5 hombres, sin diferencias significativas entre sexos $(\mathrm{p}=0,591)$. Además, la mitad $(\mathrm{n}=7)$ señaló que tenía preferencia por el donante de sexo masculino, de los cuales 3 eran pacientes mujeres. Por otro lado, 4 pacientes refirieron preferir el sexo femenino como donante, de las cuales todas eran mujeres. Los 3 pacientes restantes prefirieron no responder.

$\mathrm{Al}$ interrogar sobre si los pacientes sentían que el sexo biológico y edad del donante podría influir en ellos, un porcentaje significativamente superior $(78 \%$, $\mathrm{n}=81$ ) refirió que no, comparados con el $22 \%$ restante que respondió que sí o que no sabía $(\mathrm{p}<0,001)$. Entre los 12 pacientes que respondieron que sentían influencia, más de la mitad $(n=8)$ eran mujeres, y entre los 11 que mencionaron que no sabían, 9 fueron mujeres.

La mediana de edad de los pacientes que habían referido que no les influía el sexo biológico y la edad del donante fue significativamente superior (50,5 años; RIC: 38 - 59) comparado con aquellos que habían referido que sí les influía o que no sabían (33 años; RIC: 23 - 55) $(\mathrm{p}=0,023)$. Con respecto al sexo biológico de los pacientes entrevistados, no se observaron diferencias significativas $(\mathrm{p}=0,495)$.

De los 79 pacientes que manifestaron su interés en conocer el sexo biológico y la edad del donante, el 62\% $(\mathrm{n}=49)$ señaló que el motivo de su interés era "agradecer porque le salvó la vida o porque le dio vida" o simplemente "agradecer", mientras que el resto refirió otros motivos como "curiosidad" o "saber quién era", se observó una mayoría estadísticamente significativa entre quienes mencionaron el motivo relacionado con el agradecimiento ( $\mathrm{p}=0,0025)$. Entre estos $49 \mathrm{pa}-$ cientes, el $65 \%(n=32)$ eran mujeres, siendo este porcentaje significativamente mayor al de los hombres. $(\mathrm{p}=0,00244)$. Con respecto a la edad, tiempo en lista de espera y tiempo de trasplante, no se observaron diferencias significativas entre el grupo que refirió como motivo agradecer comparado con los que citaron otro motivo ( $\mathrm{p}=0,568 ; \mathrm{p}=0,172 ; \mathrm{p}=0,667$, respectivamente).

Al explorar sobre los temas dominantes relacionados con la necesidad de tener información sobre el donante, se extrajeron los siguientes segmentos de la entrevista: "Creo que mi donante fue una persona sana", "Mi donante era una mujer más joven porque es mejor el riñón y además las mujeres son más sanas", "Mi donante es un ángel muy bueno que me cuida y me acompaña", "Yo conozco a mi donante por medio de un sueño, donde él muere en un accidente de autos", "Mi donante era un obrero que se cayó de un andamio". En todos los casos se observa una necesidad subjetiva de construir una figura para el donante. 


\section{Discusión}

Nuestros resultados muestran que la mayoría de los pacientes que contestaron afirmativamente sobre si les interesaba saber el sexo y la edad del donante, no lo relacionaron con la posibilidad de elegir el sexo y la edad y tampoco con la creencia de que estos atributos ejercieran algún efecto sobre ellos. Sin embargo, surgió como una justificación del interés de conocer estos datos, el hecho de poder agradecer por su trasplante. Este hallazgo nos lleva a buscar una explicación de la interpretación de este agradecimiento.

Los estudios sobre el agradecimiento o gratitud se han centrado en la naturaleza de las diferencias individuales y en las consecuencias de ser una persona más o menos agradecida (Wood et al., 2007), inclusive se ha llegado a medir dicho sentimiento con escalas que evalúan las diferencias individuales en la gratitud que incluyen la frecuencia, la intensidad, la apreciación y el objeto de la gratitud (Adler \& Fagley, 2005).

Las personas que son más agradecidas tienen un mayor nivel de bienestar subjetivo, son más felices y tienen un sentimiento de satisfacción con respecto a sus vidas, con menor incidencia de depresión y estrés (Word et al., 2008; McCullough et al., 2002).

Es importante diferenciar la gratitud de la deuda moral, si bien estas dos emociones se producen después de recibir una ayuda, en el caso de la deuda moral se instala la percepción de la obligación de compensarla (Greenberg, 1980).

Este no parece ser el caso de un receptor de un trasplante ya que en general la deuda moral puede motivar a los receptores de la ayuda a evitar a la persona que les ha ayudado. En el caso de la gratitud o agradecimiento la motivación puede pasar por la búsqueda de su benefactor (Watkins et al., 2006; Tsang, 2006).

La gratitud está mucho más relacionada con la salud mental que cualquier otro rasgo de carácter. Seligman y colaboradores llevaron a cabo un estudio entre personas visitantes de un sitio web creado por el autor para tal fin, 411 adultos respondieron el cuestionario que constaba de cinco ejercicios de felicidad y un ejercicio de control con placebo. Uno de los ejercicios se centraba en construir gratitud, dos se centraron en aumentar la conciencia de lo que es más positivo sobre uno mismo, y dos centrados en identificar fortalezas de carácter. De todos estos métodos, se encontró que el que tenía mayores efectos a corto plazo provenía de una "tarjeta de gratitud", donde los participantes escribieron y entregaron una carta de agradecimiento a alguien en su vida. Este procedimiento demostró un aumento en las puntuaciones de felicidad en un $10 \%$ y una caída significativa en las puntuaciones de depresión, resultados que se prolongaron hasta un mes después de la visita (Seligman et al., 2005).

Muchos receptores sienten la necesidad de expresar su gratitud al donante, en el caso del donante vivo esta acción puede ser más fácil, pero aun en el caso del donante cadavérico la imposibilidad de no poder contactarlo no elimina el impulso de intentar agradecer, muchos receptores envían cartas de agradecimiento anónimas a las familias de los donantes a través de profesionales de la salud, en un intento de lidiar con su sentido de obligación y la mayoría encuentra esto extremadamente satisfactorio (Gill \& Lowes, 2008).

Esta forma de expresar la gratitud a través de cartas es común entre pacientes trasplantados, a modo de ejemplo, Burroughs y Selves tuvieron la idea de producir un libro de cartas de los pacientes a sus donantes, reconociendo la contribución de los mismos para salvar sus vidas y explican que un libro de cartas de los pacientes muy agradecidos sería una forma poderosa de recordar públicamente a los donantes y sus familias (Royal College of Physicians, 2010).

Un ejemplo de este libro es la carta que escribió una receptora de un trasplante cardíaco al donante y su familia, no solo agradeciendo por los órganos donados, sino también preguntando sobre quién había sido en vida y cómo había muerto (los datos con los que contaba esta paciente eran la edad y el sexo de su donante, pero también tenía información de la causa de la muerte). Esta receptora fue más allá del agradecimiento ya que se preguntó por datos particulares de su donante; como ser si había sido feliz, cómo estaba constituida su familia, cuáles eran sus gustos y qué la hacía reír. Cabe aclarar que en esa carta la paciente refiere que, al principio, no sentía como propio a su nuevo órgano y que su aceptación se produjo cuando pudo entender que su donante le había realizado un regalo, y termina la carta diciendo "mi vida, a cambio de la tuya" (BBC News, 2020).

Existen teorías que explican las reacciones psicológicas de receptores de trasplante renal y en cuanto a la internalización del órgano trasplantado. Muslin describió una de las primeras teorías que define esta apropiación del órgano como gradual; este procedimiento estaría dividido en tres estadios. En el primer estadio el órgano es percibido como un cuerpo extraño, separado del cuerpo, en el segundo los pacientes experimentan el órgano extraño cada vez más como parte de su propio cuerpo y en el tercer estadio, el órgano trasplantado se integra en la imagen corporal y se percibe como parte del propio cuerpo (Muslin, 1971). 
Muslin se basa en el concepto de que, al principio del desarrollo psíquico, el cuerpo es catectizado principalmente de manera narcisista y que no existe una mayor consideración por el mundo. Señala que, en las primeras etapas de desarrollo, el niño ya puede distinguir entre su propio cuerpo y su entorno (Muslin, 1972).

Lefebvre y Crombez enriquecieron el modelo de Muslin con observaciones sobre la relación entre paciente y donante. Estos autores argumentaron que, en una fase inicial del proceso psíquico, el paciente, mientras que experimenta el órgano trasplantado como extraño, se identifica con el donante desconocido, simplemente fantaseado. A medida que los pacientes evolucionan en el proceso psíquico durante el trasplante, el órgano se integra en su imagen corporal, mientras el donante se representa cada vez más como un objeto extraño. La representación del donante al principio refleja la autorepresentación del receptor, luego se desprende gradualmente y se mueve del núcleo del yo hacia el mundo externo, mientras que el trasplante se mueve en la dirección opuesta, desde la periferia hasta el núcleo del paciente y finalmente se integra en la autoimagen del receptor (Lefebvre \& Crombez, 1972; Lefebvre et al., 1973).

Es de señalar que en las teorías de apropiación del órgano el tiempo transcurrido desde el trasplante no ha sido considerado como una variable a destacar. En realidad, se describe que los pacientes que sienten al donante y al órgano continuamente en la etapa de cuerpo extraño tienen una forma menos madura de simbolización, por lo que en la imaginación del receptor, el órgano sigue siendo algo que pertenece al donante. Por otro lado, pacientes con una simbolización más madura tienen la capacidad de experimentar tanto el órgano como el donante situado dentro de un espacio de transición y con el tiempo integran el órgano completamente (Neukom et al., 2012).

En nuestro estudio, en los pacientes que manifestaron su interés en conocer el sexo biológico del donante con motivo de agradecer, no observamos diferencias con respecto al tiempo desde el trasplante, coincidiendo que este factor no parece estar implicado, cabe aclarar que este grupo estaba mayoritariamente integrado por mujeres, hecho que deberá ser motivo de futuras investigaciones.

La investigación de los aspectos psicológicos implicados en el trasplante de órganos y tejidos no es del todo concluyente, por lo que se requieren más estudios que logren revisar con mayor detalle las atribuciones, los significados y las experiencias que maneja el receptor durante este proceso (Fuica \& Palacios-Espinosa, 2016).
$\mathrm{Al}$ respecto, la ley de trasplante argentina prohíbe proporcionar datos personales de donantes y receptores (República Argentina, Ley 27.447 de trasplante de órganos, tejidos y células, 2018). Es de práctica habitual que ante el pedido de receptores de conocer la identidad de su donante solo se les proporcione la edad y el sexo. Pensamos que esta información podría determinar un destinatario imaginario hacia el cual va dirigido el agradecimiento, satisfaciendo la necesidad subjetiva del receptor.

Los datos obtenidos nos permiten elaborar una hipótesis respecto del trabajo psíquico que deberá afrontar el receptor del órgano trasplantado.

El paciente trasplantado inaugura una categoría previamente inexistente en cuanto impone nuevas representaciones inconscientes de la imagen del cuerpo (Doltó, 1984), de por sí nunca única ni estática, compuesta por la superposición, en la fantasía y en la realidad, de dos cuerpos humanos biológicos a través de un órgano que los vincula. El cuerpo, en la etapa del pre y postrasplante padece desajustes en su integridad y función; su protagonismo, particular y específico, es central (Duek, 2010).

La recepción de un órgano genera en el paciente múltiples emociones que se disparan al mismo tiempo, y de acuerdo con su subjetividad, encontrará distintas maneras de manifestarlo.

A partir de nuestra investigación hemos visto que la mayoría de los pacientes no elegirían el sexo biológico ni la edad del donante, descartando que recibir un órgano de sexo opuesto les generaría algún cambio en su personalidad y afectos, caso contrario a lo referido en otros estudios (Crowley-Matoka, 2005), sin embargo, los pacientes manifestaron interés por conocer el sexo y edad del donante para "agradecer".

Ante esto nos preguntamos si el conocimiento de los datos mencionados permitiría construir, en su fantasía, un guion imaginario para una representación del donante.

La fantasía tiene elementos positivos y proporciona "regresiones pequeñas y compensatorias y cumplimiento de deseos que son, en efecto reparadores" (Fenichel, 1946).

En nuestro trabajo la necesidad subjetiva del saber logra poder armar este guion imaginario con la figura del donante, por ejemplo, si el donante era joven "mi donante era una mujer más joven porque es mejor el riñón", "la causa de muerte podría ser por un accidente automovilístico", y si era mujer "seguro tenía una vida sana", y así a partir de las dos variables sexo y edad construir imaginariamente al desconocido e incorporarlo a su vida cotidiana. 
Es ahí donde creemos que surge la posibilidad del agradecimiento que perdura en el tiempo, siendo este un sentimiento de estima y reconocimiento que una persona tiene hacia quien le ha hecho un favor o prestado un servicio por el cual desea corresponderle. En el caso del trasplante de órganos cadavéricos, de no existir la construcción del donante por medio de la fantasía no habría a quien corresponderle.

Por lo que podemos concluir que el agradecimiento dirigido a esa persona, de la cual solamente se sabe el sexo y la edad, permitiría al paciente poder realizar una construcción imaginaria del donante, cerrando de alguna manera el circuito que se genera por la donación, la recepción del órgano y la mejoría de la calidad de vida.

\section{Conflictos de intereses: los autores declaran no tener} conflictos de intereses.

\section{Referencias bibliográficas}

Adler, M.G., Fagley, N.S. (2005). Appreciation: Individual differences in findingvalue and meaning as a unique predictor of subjective well-being. Journal of Personality, 73(1), 79-114. https://doi.org/10.1111/j.14676494.2004.00305.x

BBC News https://www.bbc.com/mundo/noticias/2010/11/101111_donante_organos_agradecimiento. Último acceso: 11 de diciembre de 2020.

Crowley-Matoka, M. (2005). Desperately Seeking "Normal": the Promise and Perilsof Living with Kidney Transplantation. Social Science \& Medicine, 61(4), 821-831. 10.1016/j.socscimed.2004.08.043

Doltó F. (1984). La imagen inconsciente del cuerpo. Éditions du Seuil.

Duek, R. (2010). El cuerpo antes y después de un trasplante de órgano. Una mirada psicoanalítica. Psicoanálisis, 32(2-3), 255-60.

Fenichel, O. (1946). The Psychoanalytic Theory of Neurosis. Norton \& Company, p. 554.

Fuica, S., Palacios-Espinosa, X. (2016). Significados atribuidos por el paciente trasplantado al órgano recibido, al donante, a la vida y a la muerte. Pensamiento Psicológico, 14(2), 19-32. doi:10.11144/Javerianacali.PPSI142.sapt

Gill, P., Lowes, L. (2008). Gift exchange and organ donation: donor and recipient experiences of live related kidney transplantation. International Journal of Nursing Studies, 45(11), 1607-1617.doi: 10.1016/j.ijnurstu.2008.03.004.

Greenberg, M. S. (1980). A theory of in debtedness. En K. J. Gergen, M.S. Greenberg \& R.H. Wills (Eds.), Social exchange: Advances in theory and research. Plenum.
Lefebvre, P., Crombez, J. C., LeBeuf, J. (1973). Psychological dimension and psychopathological potential facquiring a kidney. Can Psychiatr Assoc $J, 18: 495-500$.

Lefebvre, P., Crombez, J.C. (1972). The behavioural responses of renal transplant patients as seen throught heir fantasy life. Can J Psychiatr 17:19-23. https://doi.org/10.1177/07067437720176S205

McCullough, M.E., Emmons, R.A., \& Tsang, J. (2002). The grateful disposition: A conceptual and empirical topography. Journal of Personality and Social Psychology, 82(1), 112-127. https://doi.org/10.1037/0022$\underline{3514.82 .1 .112}$

Muslin H. L. (1972). The emotional response to the kidney transplant: the process of internalization. Can Psychiatr Assoc J, 17(2):SS 2. doi: $\underline{10.1177 / 07067437720176 s 202}$

Muslin, H. L. (1971). On acquiring a kidney. Am J Psychiatry, 127(9), 11858. doi: 10.1176/ajp.127.9.1185

Neukom, M., Corti, V., Boothe, B., Boehler, A., \& Goetzmann, L. (2012). Fantasized recipient-donor relationships following lung transplantations: a qualitative case analysis based on patient narratives. Int J Psychoanal, 93(1),117-37. doi:10.1111/j.1745-8315.2011.00496.x

Organización Mundial de la Salud. (1946). Constitución de la Organización Mundial de la Salud. Acte 2, 100. https://www.who.int/governance/ eb/who_constitution_sp.pdf Último acceso: 20 de diciembre de 2020.

República Argentina. Ley 27.447 de trasplante de órganos, tejidos y células, 26/07/2018. https://www.argentina.gob.ar/sites/default/files/ley-27447.pdfÚltimo acceso: 20 de diciembre de 2020

Royal College of Physicians. (2010). Thank You for Life: Letters from Transplant Recipients to Donors' Families. Royal College of Physicians of London.

Schiavelli, R. O., Fontana, R. S., Merino, D. O. (2011). Aspectos sociales de la no adherencia al tratamiento en trasplante renal. Biophronesis 6(2), 1-13. www.fmv-uba.org.ar> revistasylibrosdigitales > antropología > Schiavelli. Último acceso: 11 de diciembre de 2020

Seligman, M. E. P., Steen, T.A., Park, N., \& Peterson, C. (2005). Positive psychology progress: Empirical validation of interventions. American Psychologist, 60(5), 410-421. https://doi.org/10.1037/0003-066X.60.5.410

Tsang, J-A. (2006). Gratitude and prosocial behaviour: An experimental test of gratitude. Cognition and Emotion, 20(1), 138-148. https://doi. org/10.1080/02699930500172341

Vamos, M. (2010). Organ transplantation and magical thinking. Aust NZ J Psychiatry, 44(10), 883-7. doi: 10.3109/00048674.2010.498786

Watkins, P.C., Scheer, J., Ovnicek, M., \& Kolts, R. (2006). The debt of gratitude: Dissociating gratitude and indebtedness. Cognition and Emotion, 20(2), 217-241. https://doi.org/10.1080/02699930500172291

Wood, A., Stephen, J., Linley, A. (2007). Gratitude-Parent of All Virtues. The Psychologist 20(1), 18-21.

Wood, A.M., Joseph, S., Maltby, J. (2008). Gratitud euniquely predicts satisfaction with life: Incremental validity above the domains and facet sof the five factor model. Personality and Individual Differences, 45(1), 49-54. 